Proceedings of the Edinburgh Mathematical Society (2003) 46, 421-433 (C)

DOI:10.1017/S0013091501000980 Printed in the United Kingdom

\title{
QUANTUM CHANNELS, WAVELETS, DILATIONS AND REPRESENTATIONS OF $\mathcal{O}_{n}$
}

\author{
DAVID W. KRIBS* \\ Department of Mathematics and Statistics, University of Guelph, Guelph, \\ Ontario N1G2W1, Canada (kribs@math.purdue.edu)
}

(Received 28 October 2001)

\begin{abstract}
We show that the representations of the Cuntz $C^{*}$-algebras $\mathcal{O}_{n}$ which arise in wavelet analysis and dilation theory can be classified through a simple analysis of completely positive maps on finitedimensional space. Based on this analysis, we find an application in quantum information theory; namely, a structure theorem for the fixed-point set of a unital quantum channel. We also include some open problems motivated by this work.
\end{abstract}

Keywords: operator; completely positive map; quantum channel; orthogonal wavelet; isometric dilation; Cuntz algebra

2000 Mathematics subject classification: Primary 46L45; 47A20; 46L60; 42C40; 81P15

There has been considerable recent interest in the analysis of completely positive maps on finite-dimensional space. There are a number of reasons for this including connections with wavelet analysis $[\mathbf{3}, \mathbf{4}, \mathbf{1 5}]$, dilation theory $[\mathbf{1 1}, \mathbf{1 6}]$, representation theory of the Cuntz $C^{*}$-algebras $\mathcal{O}_{n}[\mathbf{3}, \mathbf{4}, \mathbf{1 0}, \mathbf{1 1}]$, and quantum information theory $[\mathbf{1}, \mathbf{1 7}, \mathbf{2 1}, \mathbf{2 2}]$. The results obtained in the current paper have implications for each of these areas. In presenting this work, another goal we have is to push further the connections between the various areas mentioned above.

In $\S 1$ we establish a result for completely positive maps. While we focus on the finitedimensional setting, this is not necessary in the proof. A structure theorem for the fixedpoint set of a unital quantum channel is contained in $\S 2$. In particular, we prove that the fixed-point set is a $C^{*}$-algebra which is equal to the commutant of the algebra generated by any choice of row contraction which determines the channel. We discuss the twodimensional channels $[\mathbf{1 7}, \mathbf{2 1}]$, and use the theorem to classify them by their fixed-point sets. The representation theory for $\mathcal{O}_{n}$ is considered in $\S 3$. We focus on a subclass of representations arising in dilation theory and wavelet analysis $[\mathbf{3}, \mathbf{4}, \mathbf{1 0}, \mathbf{1 1}, \mathbf{1 5}]$. Each of these representations determines a completely positive map on finite-dimensional space. We ask if these representations can be classified just by examining the map. An affirmative answer is provided by the result on completely positive maps from the first section. Finally, in $\S 4$ we pose some open questions which are motivated by this work.

* Present address: Department of Mathematics, Purdue University, West Lafayette, IN 47907, USA. 


\section{Completely positive maps}

In this section we present a theorem for completely positive maps on finite-dimensional space. Let $\mathcal{K}$ be a finite-dimensional Hilbert space and let $\mathcal{B}(\mathcal{K})$ be the bounded operators on $\mathcal{K}$. It is well known (see, for example, $[\mathbf{7}, \mathbf{1 8}, \mathbf{1 9}]$ ) that every completely positive map $\Phi: \mathcal{B}(\mathcal{K}) \rightarrow \mathcal{B}(\mathcal{K})$ is determined by a row matrix $A=\left(A_{1}, \ldots, A_{n}\right)$ of operators in $\mathcal{B}(\mathcal{K})$ in the following sense:

$$
\Phi(X)=\sum_{i=1}^{n} A_{i} X A_{i}^{*} \quad \text { for } X \in \mathcal{B}(\mathcal{K}) .
$$

The map is unital if, in addition, $\Phi(I)=\sum_{i=1}^{n} A_{i} A_{i}^{*}=A A^{*}=I$. Of course, there will be many choices of tuples $A$ which determine a given completely positive map in this way. We prove the following result.

Theorem 1.1. Let $\mathcal{K}$ be a finite-dimensional Hilbert space. Let $\Phi: \mathcal{B}(\mathcal{K}) \rightarrow \mathcal{B}(\mathcal{K})$ be a completely positive unital map, and suppose $A=\left(A_{1}, \ldots, A_{n}\right)$ is a choice of operators which determine $\Phi$ as in (1.1). If $p$ is an orthogonal projection in $\mathcal{B}(\mathcal{K})$, then we have the following equivalences for the range space $\operatorname{Ran}(p)$ of the projection.

(i) $\Phi(p) \geqslant p$ if and only if $\operatorname{Ran}(p)$ is $A_{i}^{*}$-invariant for $1 \leqslant i \leqslant n$.

(ii) $\Phi(p) \leqslant p$ if and only if $\operatorname{Ran}(p)$ is $A_{i}$-invariant for $1 \leqslant i \leqslant n$.

(iii) $\Phi(p)=p$ if and only if $\operatorname{Ran}(p)$ is $A_{i}$-reducing for $1 \leqslant i \leqslant n$.

Furthermore, for a given projection $p$, one of these equivalences holds for a particular choice of $A$ in (1.1) if and only if it holds for all choices of $A$ in (1.1).

In each of these cases the limit

$$
\Phi^{\infty}(p):=\lim _{k \rightarrow \infty} \Phi^{k}(p)
$$

exists. This operator belongs to the fixed-point set $\mathcal{B}(\mathcal{K})^{\Phi}$ of $\Phi$, and it is computed from the fixed-point set as follows.

(i) $^{\prime}$ If $\Phi(p) \geqslant p$, then $\Phi^{\infty}(p)=\inf \left\{X \in \mathcal{B}(\mathcal{K})^{\Phi}: X \geqslant p\right\}$.

(ii) $^{\prime}$ If $\Phi(p) \leqslant p$, then $\Phi^{\infty}(p)=\sup \left\{X \in \mathcal{B}(\mathcal{K})^{\Phi}: X \leqslant p\right\}$.

(iii) $^{\prime}$ If $\Phi(p)=p$, then the infimum and supremum from (i) ${ }^{\prime}$ and (ii) ${ }^{\prime}$ are both equal to $p$.

While the existence of the limit $\Phi^{\infty}(p)$ and the final three facts seem to be of independent interest, the applications of this result contained in the rest of the paper are based on (i)-(iii).

Note 1.2. After preparing this article the author discovered that conditions (ii) and (iii) were recently established in [5], although the proofs here are different.

Before proving the theorem, we establish a lemma which generalizes a result from $[\mathbf{1 1}]$. 
Lemma 1.3. Let $X$ be a positive operator in $\mathcal{B}(\mathcal{K})$ which satisfies the inequality $0 \leqslant X \leqslant \Phi(X)$. Then the eigenspace $\operatorname{ker}(X-\|X\| I)$ is $A_{i}^{*}$-invariant for $1 \leqslant i \leqslant n$.

Proof. Without loss of generality assume that $\|X\|=1$. Let $\mathcal{M}=\operatorname{ker}(X-I)$. Then for any vector $\xi \in \mathcal{M}$,

$$
\begin{aligned}
\|\xi\|^{2}=(X \xi, \xi) & \leqslant \sum_{i=1}^{n}\left(A_{i} X A_{i}^{*} \xi, \xi\right) \\
& =\sum_{i=1}^{n}\left(X A_{i}^{*} \xi, A_{i}^{*} \xi\right) \leqslant \sum_{i=1}^{n}\left(A_{i}^{*} \xi, A_{i}^{*} \xi\right)=\|\xi\|^{2} .
\end{aligned}
$$

In particular, all the inner product inequalities are actually equalities. Since $X$ is a positive contraction, the only way this can happen is if each of the vectors $A_{i}^{*} \xi$ belongs to $\mathcal{M}$. Hence $\mathcal{M}$ is $A_{i}^{*}$-invariant.

Proof of Theorem 1.1. First note that the equivalences (i) and (ii) are duals of each other. Indeed, since $\Phi$ is unital,

$$
\Phi(p) \geqslant p \quad \text { if and only if } \Phi(I-p) \leqslant I-p,
$$

and the subspace $\operatorname{Ran}(p)$ is $A_{i}^{*}$-invariant precisely when $\operatorname{Ran}(I-p)=\operatorname{Ran}(p)^{\perp}$ is $A_{i^{-}}$ invariant. Thus we shall prove (i).

If $\Phi(p) \geqslant p$, then an application of the previous lemma yields the $A_{i}^{*}$-invariance of $\operatorname{Ran}(p)$. To see the converse of (i), with respect to the orthogonal decomposition $\mathcal{K}=$ $p \mathcal{K} \oplus p^{\perp} \mathcal{K}$, suppose $A_{i}$ can be written in matrix form as

$$
A_{i}=\left[\begin{array}{cc}
B_{i} & 0 \\
C_{i} & D_{i}
\end{array}\right] \quad \text { for } 1 \leqslant i \leqslant n
$$

Then the relation $\Phi\left(I_{\mathcal{K}}\right)=\sum_{i=1}^{n} A_{i} A_{i}^{*}=I_{\mathcal{K}}$ yields the identities

$$
\sum_{i=1}^{n} B_{i} B_{i}^{*}=I_{p \mathcal{K}}, \quad \sum_{i=1}^{n} B_{i} C_{i}^{*}=0 \quad \text { and } \quad \sum_{i=1}^{n}\left(C_{i} C_{i}^{*}+D_{i} D_{i}^{*}\right)=I_{p^{\perp} \mathcal{K}} .
$$

Thus upon writing

$$
p=\left[\begin{array}{ll}
I & 0 \\
0 & 0
\end{array}\right]
$$

with respect to this spatial decomposition we get

$$
\Phi(p)=\sum_{i=1}^{n} A_{i} p A_{i}^{*}=\left[\begin{array}{cc}
I & 0 \\
0 & \sum_{i=1}^{n} C_{i} C_{i}^{*}
\end{array}\right] \geqslant\left[\begin{array}{ll}
I & 0 \\
0 & 0
\end{array}\right]=p .
$$

For (iii), notice that the previous computation shows that $\Phi(p)=p$ when $\operatorname{Ran}(p)=p \mathcal{K}$ is $A_{i}$-reducing. Indeed, the subspace $p \mathcal{K}$ is reducing for the operators $A=\left(A_{1}, \ldots, A_{n}\right)$ 
exactly when $C_{i}=0$ for $1 \leqslant i \leqslant n$. On the other hand, if $\Phi(p)=p$, then the inequalities in (i) and (ii) are satisfied. Hence $\operatorname{Ran}(p)$ is $A_{i}$-reducing.

Concerning the limits, suppose $p$ satisfies equivalence (i). Since $\Phi$ is positive and unital, we have

$$
0 \leqslant p \leqslant \Phi(p) \leqslant \Phi^{2}(p) \leqslant \cdots \leqslant \Phi^{k}(p) \leqslant \cdots \leqslant I .
$$

This is a monotone increasing sequence of positive operators which is bounded above, hence we obtain the existence of the limit $\Phi^{\infty}(p)=\lim _{k \rightarrow \infty} \Phi^{k}(p)$. It is clear from the form (1.1) that $\Phi$ is continuous, hence $\Phi^{\infty}(p)$ is fixed under the action of $\Phi$. On the other hand, when $X \geqslant p$ is fixed by the map, we have $X \geqslant \Phi^{k}(p)$ for $k \geqslant 1$, showing that $\Phi^{\infty}(p)$ is bounded above by every fixed point which majorizes $p$. It follows that $\Phi^{\infty}(p)$ actually is the infimum.

The proof of (ii)' is analogous since, in that case, the operators $\Phi^{k}(p)$ form a decreasing sequence of positive operators which are majorized by $p$. Finally, when $\Phi(p)=p$, we get $\Phi^{\infty}(p)=p$ so that (i)' and (ii)' show that the infimum and supremum are both equal to $p$.

Remark 1.4. While our focus is on the finite-dimensional case, we note that the proof of Theorem 1.1 works for any completely positive unital map determined as in (1.1) by a row contraction $A$. The only change in the conclusion is that the limit $\Phi^{\infty}(p)$ converges in the strong operator topology.

\section{Quantum channels}

Mathematically, a quantum channel is a completely positive trace-preserving map on finite-dimensional space. In the language of quantum information theory, a channel describes the transfer of quantum information, or qubits, from 'Alice' to 'Bob' (see $[\mathbf{1}, \mathbf{1 7}, \mathbf{2 1}, \mathbf{2 2}]$ for some recent related analysis, as well as [8] for general information). The operators $A=\left(A_{1}, \ldots, A_{n}\right)$ which determine a quantum channel $\Phi$ as in (1.1) are called the Kraus operators $[\mathbf{1 8}]$ of the channel.

In general, the fixed-point set $\mathcal{B}(\mathcal{K})^{\Phi}=\{X \in \mathcal{B}(\mathcal{K}) \mid \Phi(X)=X\}$ of a completely positive map is just a self-adjoint subspace. In particular, generally the fixed-point set is not closed under multiplication. We obtain the following structure theorem for the fixed-point set of a unital quantum channel.

Theorem 2.1. Let $\Phi: \mathcal{B}(\mathcal{K}) \rightarrow \mathcal{B}(\mathcal{K})$ be a unital quantum channel. If $A=$ $\left(A_{1}, \ldots, A_{n}\right)$ determines $\Phi$ as in (1.1), then the algebra $\mathfrak{A}$ generated by $A_{1}, \ldots, A_{n}$ is a $*$-algebra which depends only on $\Phi$. Further, the fixed-point set $\mathcal{B}(\mathcal{K})^{\Phi}$ of the map $\Phi$ coincides with the commutant of $\mathfrak{A}, \mathfrak{A}^{\prime}=\left\{X \in \mathcal{B}(\mathcal{K}) \mid A_{i} X=X A_{i}\right.$ for $\left.1 \leqslant i \leqslant n\right\}$, and hence is itself a $*$-algebra containing the identity operator on $\mathcal{K}$.

We begin by pointing out a special case of the theorem, established previously in $[\mathbf{5}, \mathbf{1 1}]$, which holds more generally. For completeness we provide a proof.

Lemma 2.2. Let $\mathcal{K}$ be a finite-dimensional space and let $\Phi: \mathcal{B}(\mathcal{K}) \rightarrow \mathcal{B}(\mathcal{K})$ be a unital completely positive map which is determined as in (1.1) by $A=\left(A_{1}, \ldots, A_{n}\right)$. If $\mathfrak{A}=\mathcal{B}(\mathcal{K})$, then the fixed-point set for $\Phi$ consists of scalars, $\mathcal{B}(\mathcal{K})^{\Phi}=\mathbb{C} I$. 
Proof. Suppose $X=X^{*}$ is non-scalar and satisfies $\Phi(X)=X$. Then Lemma 1.3 can be adapted to show that the eigenspaces corresponding to the two extremal eigenvalues for $X$ are perpendicular $A_{i}^{*}$-invariant subspaces, which are both non-trivial since $X$ is non-scalar. Thus the algebra $\mathfrak{A}^{*}$ has proper invariant subspaces, a contradiction.

The key observation for unital quantum channels is that invariant subspaces for determining $n$-tuples are actually reducing.

Lemma 2.3. Let $\Phi: \mathcal{B}(\mathcal{K}) \rightarrow \mathcal{B}(\mathcal{K})$ be a unital quantum channel. Then every projection which satisfies $\Phi(p) \geqslant p$ also satisfies $\Phi(p)=p$. Thus if $A=\left(A_{1}, \ldots, A_{n}\right)$ determines $\Phi$, then every subspace which is invariant for the family $\left\{A_{i}^{*}: 1 \leqslant i \leqslant n\right\}$ is also reducing for the family. Furthermore, every subspace which is invariant for $\left\{A_{i}: 1 \leqslant i \leqslant n\right\}$ is also reducing.

Proof. Since $\Phi$ is positive and unital, we have $0 \leqslant \Phi(p) \leqslant I$. Thus if we are given a projection $p$ which satisfies $p \leqslant \Phi(p)$, then trace preservation ensures we must have equality, $p=\Phi(p)$. A similar analysis follows for the dual notion $\Phi(p) \leqslant p \leqslant I$. The rest of the lemma follows from Theorem 1.1.

Proof of Theorem 2.1. We first show that $\mathfrak{A}$ is a $*$-algebra. Let $\left\{p_{j}\right\}$ be a maximal family of pairwise orthogonal projections which are each minimal reducing for the family $\left\{A_{i}: 1 \leqslant i \leqslant n\right\}$. Let $\mathcal{K}_{j}=p_{j} \mathcal{K}$ for each $j$. Then $A_{i} p_{j}=p_{j} A_{i}$ for all $i, j$, and by maximality $I_{\mathcal{K}}=\sum_{j} p_{j}$. Hence the algebra $\mathfrak{A}=\sum_{j} p_{j} \mathfrak{A} p_{j}$ is block diagonal with respect to this family, and the blocks $\mathfrak{A} p_{j}=p_{j} \mathfrak{A}=p_{j} \mathfrak{A} p_{j}$ are algebras themselves. But the trivial subspaces are the only subspaces which are invariant for $\mathfrak{A} p_{j}$. Indeed, if a subspace $p \mathcal{K}$ is invariant for each $A_{i}$ and $p \leqslant p_{j}$ is supported on some $p_{j}$, then $p \mathcal{K}$ is reducing for the $A_{i}$ by Lemma 2.3, and hence by the minimality of $p_{j}$ we have either $p=0$ or $p=p_{j}$. It follows from Burnside's classical theorem that the restricted finite-dimensional algebra $\mathfrak{A} p_{j}$ is equal to $\mathcal{B}\left(\mathcal{K}_{j}\right)$, in particular the algebras $\mathfrak{A} p_{j}$ are self-adjoint. Therefore, $\mathfrak{A}=\mathfrak{A}^{*}$ is a $*$-algebra. (In particular, it is a finite-dimensional $C^{*}$-algebra.)

Since $\Phi$ is unital, that is $\Phi(I)=I$, it is clear that every $X$ which commutes with $A_{1}, \ldots, A_{n}$ will be fixed by $\Phi$. Hence the fixed-point set $\mathcal{B}(\mathcal{K})^{\Phi}$ contains the commutant $\mathfrak{A}^{\prime}$.

To see the converse, let $A_{i, j}=A_{i} p_{j}$ for all $j$ and $i=1, \ldots, n$, and for each $j$ put $B_{j}=$ $\left(A_{1, j}, \ldots, A_{n, j}\right)$. Given $X \in \mathcal{B}(\mathcal{K})$, let $X=\left(X_{j k}\right)$, where $X_{j k}=p_{j} X p_{k}$. If $X$ satisfies $\Phi(X)=X$, then a computation shows that $\Phi_{j}\left(X_{j j}\right)=X_{j j}$, where $\Phi_{j}: \mathcal{B}\left(\mathcal{K}_{j}\right) \rightarrow \mathcal{B}\left(\mathcal{K}_{j}\right)$ is the unital completely positive map $\Phi_{j}(Y)=\sum_{i=1}^{n} A_{i, j} Y A_{i, j}^{*}$. However, as observed above, the minimality of $p_{j}$ as an $A_{i}$-invariant subspace gives us $\mathfrak{A} p_{j}=\mathcal{B}\left(\mathcal{K}_{j}\right)$. Hence by Lemma 2.2, we have $X_{j j}=x_{j j} p_{j}$ for some scalar $x_{j j}$.

For $j \neq k$, we claim that either $X_{j k}=0=X_{k j}$ for all $X=X^{*}$ in $\mathcal{B}(\mathcal{K})^{\Phi}$, or there is a unitary $W_{j k}: \mathcal{K}_{k} \rightarrow \mathcal{K}_{j}$ with $A_{i, j}=W_{j k} A_{i, k} W_{j k}^{*}$ for $i=1, \ldots, n$. Thus suppose there is an $X=X^{*}$ with $\Phi(X)=X$ and $X_{j k} \neq 0$, normalized so that $\left\|X_{j k}\right\|=1$. Let $\mathcal{M}=\left\{\xi \in \mathcal{K}_{k}:\left\|X_{j k} \xi\right\|=\|\xi\|\right\}$, and let $\mathcal{N}=X_{j k} \mathcal{M}$ be the corresponding subspace of $\mathcal{K}_{j}$. Then for $\xi \in \mathcal{M}$ we have

$$
X_{j k} \xi=\left(p_{j} \Phi(X) p_{k}\right) \xi=\Phi\left(X_{j k}\right) \xi=\left(B_{j} X_{j k}^{(n)} B_{k}^{*}\right) \xi
$$


This implies that each $A_{i, k}^{*}$ leaves $\mathcal{M}$ invariant. Indeed, since $B_{j}$ and $B_{k}^{*}$ are contractions, and $X_{j k}$ achieves its norm on $\xi$, it follows that $B_{k}^{*} \xi$ belongs to the subspace $\mathcal{M}^{(n)}$ on which $X_{j k}^{(n)}$ achieves its norm. Thus by Lemma $2.3, \mathcal{M}$ is an $A_{i}$-reducing subspace contained in $\mathcal{K}_{k}$. Since $\mathcal{M}$ is non-zero, the minimality of $p_{k}$ gives $\mathcal{M}=\mathcal{K}_{k}$. By considering $X_{k j}=X_{j k}^{*}$, we see that $\mathcal{N}=\mathcal{K}_{j}$ also. In particular, $X_{j k}$ and $X_{j k}^{*}$ are partial isometries, and it follows that $W_{j k}=\left.X_{j k}\right|_{\mathcal{K}_{k}}: \mathcal{K}_{k} \rightarrow \mathcal{K}_{j}$ is a unitary operator.

The identity above shows that $W_{j k}=B_{j} W_{j k}^{(n)} B_{k}^{*}$. Hence, for $\xi \in \mathcal{K}_{k}$,

$$
\|\xi\|=\left\|W_{j k} \xi\right\|=\left\|B_{j} W_{j k}^{(n)} B_{k}^{*} \xi\right\| \leqslant\left\|W_{j k}^{(n)} B_{k}^{*} \xi\right\| \leqslant\|\xi\| .
$$

Thus $B_{j}$ acts as an isometry from the range $\operatorname{Ran} W_{j k}^{(n)} B_{k}^{*}$ onto the range $\operatorname{Ran} W_{j k}=\mathcal{K}_{j}$. As $B_{j}$ is a row contraction, it must be zero on the orthogonal complement of $\operatorname{Ran} W_{j k}^{(n)} B_{k}^{*}$. Hence, $B_{j}^{*}$ is an isometry from $\mathcal{K}_{j}$ onto $\operatorname{Ran} W_{j k}^{(n)} B_{k}^{*}$. Consequently, $B_{j}^{*} W_{j k}=W_{j k}^{(n)} B_{k}^{*}$; in other words, $A_{i, j}^{*}=W_{j k} A_{i, k}^{*} W_{j k}^{*}$ for $i=1, \ldots, n$, as claimed.

Now suppose $\Phi(Y)=Y=Y^{*}=\left(Y_{j k}\right)$. If $j \neq k$ is a pair which fits into the above analysis, then

$$
\begin{aligned}
W_{j k} \Phi_{k}\left(W_{j k}^{*} Y_{j k}\right) & =\sum_{i=1}^{n} W_{j k} A_{i, k} W_{j k}^{*} Y_{j k} A_{i, k}^{*} \\
& =\sum_{i=1}^{n} A_{i, j} Y_{j k} A_{i, k}^{*} \\
& =p_{j} \Phi(Y) p_{k}=Y_{j k} .
\end{aligned}
$$

Thus by Lemma 2.2, $W_{j k}^{*} Y_{j k}$ is scalar. Hence $Y_{j k}=y_{j k} W_{j k}$ for some scalar $y_{j k}$, and also $Y_{k j}=Y_{j k}^{*}=\bar{y}_{j k} W_{j k}^{*}$. The other off-diagonal entries of $Y$ are either zero or have a similar form.

This analysis gives us a handle on the matrix entries in the decomposition $X=\left(X_{j k}\right)$ for $X=X^{*}$ in $\mathcal{B}(\mathcal{K})^{\Phi}$. The corresponding form for each $A_{i}$ in this decomposition is given by $A_{i}=\sum_{j} A_{i} p_{j}=\sum_{j} A_{i, j}$, and hence a computation shows that $X A_{i}=A_{i} X$ for $i=1, \ldots, n$ and $X=X^{*}$ in $\mathcal{B}(\mathcal{K})^{\Phi}$. Since the self-adjoint subspace $\mathcal{B}(\mathcal{K})^{\Phi}$ is spanned by its self-adjoint part, it follows that $\mathfrak{A}^{\prime}$ contains the entire fixed-point set $\mathcal{B}(\mathcal{K})^{\Phi}$. This completes the proof.

Note 2.4. We note that our approach in proving Theorem 2.1 was motivated by work in $[\mathbf{1 1}]$ on dilation theory, which is discussed in the next section. This connection leads to an open question posed in $\S 4$ which, if answered, could mesh the theory of quantum channels with that of certain operator algebras on infinite-dimensional space.

We finish this section by showing how this theorem can be used to classify quantum channels by their fixed-point sets.

Example 2.5. The quantum channels on $\mathcal{M}_{2}=\mathcal{B}\left(\mathbb{C}^{2}\right)$, the so-called qubit channels, were recently characterized in $[\mathbf{2 1}]$. The identity matrix together with the Pauli matrices 
$\left\{I, \sigma_{x}, \sigma_{y}, \sigma_{z}\right\}$ form a basis for $\mathcal{M}_{2}$, where

$$
\sigma_{x}=\left(\begin{array}{cc}
0 & 1 \\
1 & 0
\end{array}\right), \quad \sigma_{y}=\left(\begin{array}{cc}
0 & -\mathrm{i} \\
\mathrm{i} & 0
\end{array}\right), \quad \sigma_{z}=\left(\begin{array}{cc}
1 & 0 \\
0 & -1
\end{array}\right)
$$

Every unital qubit channel is equivalent, through unitary conjugations at both the input and output stages, to a map $\Phi$ which is diagonal and real with respect to this basis. In other words, $\Phi(I)=I, \Phi\left(\sigma_{x}\right)=\lambda_{1} \sigma_{x}, \Phi\left(\sigma_{y}\right)=\lambda_{2} \sigma_{y}, \Phi\left(\sigma_{z}\right)=\lambda_{3} \sigma_{z}$, where $\lambda_{1}, \lambda_{2}, \lambda_{3} \in \mathbb{R}$ (for more on the geometry of channels see $[8]$ ).

Thus describing the set of channels on $\mathcal{M}_{2}$ amounts to deriving conditions on the $\lambda_{k}$ which guarantee such a diagonal map is completely positive and trace preserving. The norm of a completely positive map $\Phi$ is given by $\|\Phi\|=\|\Phi(I)\|$. Hence for these diagonal maps, a simple necessary condition for complete positivity is that each $\left|\lambda_{k}\right| \leqslant 1$. Using Choi's Lemma [7], necessary and sufficient conditions on the $\lambda_{k}$ were computed in [21] which lead to a description of the entire set of qubit channels, though the conditions are rather technical.

From Theorem 2.1 and the basic theory of finite-dimensional $C^{*}$-algebras, we know there are just three possibilities for the fixed-point algebra $\mathcal{M}_{2}^{\Phi}$ of a unital qubit channel $\Phi$. Somewhat surprisingly, we do not need the extra conditions on $\left\{\lambda_{1}, \lambda_{2}, \lambda_{3}\right\}$ from $[\mathbf{2 1}]$ to classify the fixed-point sets.

Corollary 2.6. Let $\Phi: \mathcal{M}_{2} \rightarrow \mathcal{M}_{2}$ be a unital quantum channel. Then the fixed-point set for $\Phi$ is a $*$-subalgebra of $\mathcal{M}_{2}$ containing the identity operator and satisfying one of the following conditions.

(i) $\mathcal{M}_{2}^{\Phi}=\mathcal{M}_{2}$ if and only if each $\lambda_{k}=1$ and $\Phi$ is the identity map.

(ii) $\mathcal{M}_{2}^{\Phi}=\mathbb{C} I$ if and only if $\lambda_{k} \neq 1$ for $k=1,2,3$.

(iii) $\mathcal{M}_{2}^{\Phi}=\operatorname{span}\left\{\left|v_{1}\right\rangle\left\langle v_{1}|,| v_{2}\right\rangle\left\langle v_{2}\right|\right\}$, where $\left\{v_{1}, v_{2}\right\}$ is an orthonormal basis for $\mathbb{C}^{2}$ and $\left|v_{i}\right\rangle\left\langle v_{i}\right|$ is the rank-one projection of $\mathbb{C}^{2}$ onto $\operatorname{span}\left\{v_{i}\right\}$, and this case holds if and only if exactly one of $\left\{\lambda_{1}, \lambda_{2}, \lambda_{3}\right\}$ is equal to 1 .

Proof. By the previous discussion we may assume $\Phi$ is diagonal with respect to the Pauli basis and that $\left|\lambda_{k}\right| \leqslant 1$ for $k=1,2,3$. An elementary computation shows that the Pauli basis has the property that projections $p=p^{2}=p^{*} \in \mathcal{M}_{2}$ are either trivial $(p=0$ or $p=I$ ) or represented as

$$
p=\frac{1}{2} I+a \sigma_{x}+b \sigma_{y}+c \sigma_{z}
$$

where $a, b, c \in \mathbb{R}$ and $a^{2}+b^{2}+c^{2}=\frac{1}{4}$. Thus $\Phi(p)=p$ exactly when $a=a \lambda_{1}, b=b \lambda_{2}$ and $c=c \lambda_{3}$.

In particular, the case $\lambda_{1}=\lambda_{2}=\lambda_{3}=1$ corresponds to $a, b, c$ being free variables and $\Phi$ being the identity map on $\mathcal{M}_{2}$. By Theorem 2.1 the operator algebraic characterizations of $\mathcal{M}_{2}^{\Phi}$ in (ii) and (iii) are the only remaining possibilities for the fixed-point set. Further, the finite-dimensional $C^{*}$-algebra $\mathcal{M}_{2}^{\Phi}$ is spanned by its projections. Thus $\mathcal{M}_{2}^{\Phi}=\mathbb{C} I$ 
exactly when there are no non-trivial projections fixed by $\Phi$. Equivalently, there are no solutions $a, b, c$ to the above identities. Clearly, this holds if and only if each $\lambda_{k} \neq 1$.

Hence the remaining cases must satisfy (iii), and we claim this is when exactly one of the $\lambda_{k}$ is equal to 1 . Indeed, either one or two of the $\lambda_{k}$ must be equal to 1 for the case (iii) maps, since otherwise we would be in one of the first two cases by the previous paragraph. If, say, $\lambda_{1}=\lambda_{2}=1$, then $\Phi$ fixes $\sigma_{x}$ and $\sigma_{y}$. Whence, $(-\mathrm{i}) \sigma_{x} \sigma_{y}=\sigma_{z}$ is also fixed by $\Phi$ since the fixed-point set is an algebra. But this would imply that $\lambda_{3}=1$, and we are really in case (i). Similarly, it is easy to see that when any two of $\left\{\lambda_{1}, \lambda_{2}, \lambda_{3}\right\}$ are equal to 1 , the third must be as well. Thus the operator algebra characterization of $\mathcal{M}_{2}^{\Phi}$ in (iii) occurs precisely when there is exactly one $\lambda_{k}$ equal to 1 .

\section{Applications to representation theory for $\mathcal{O}_{n}$}

In this section we show that representations of the Cuntz $C^{*}$-algebra $\mathcal{O}_{n}$ arising from dilations and wavelets can be classified through an analysis of completely positive maps. Given a positive integer $n \geqslant 2, \mathcal{O}_{n}$ is the universal $C^{*}$-algebra generated by the relations

$$
s_{i}^{*} s_{j}=\delta_{i j} I \quad \text { for } 1 \leqslant i, j \leqslant n \text { and } \sum_{i=1}^{n} s_{i} s_{i}^{*}=I .
$$

An $n$-tuple $S=\left(S_{1}, \ldots, S_{n}\right)$ of operators in $\mathcal{B}(\mathcal{H})$ which satisfies these relations consists of isometries with pairwise orthogonal ranges, for which the range projections of the isometries span the entire (necessarily infinite-dimensional) Hilbert space $\mathcal{H}$. Up to isomorphism, $\mathcal{O}_{n}$ is the $C^{*}$-algebra generated by any such $n$-tuple, since it is simple. A theorem of Glimm's [13] suggests it is not possible to find a meaningful classification of all representations of $\mathcal{O}_{n}$ (it is an 'NGCR' algebra). However, there are good reasons for attempting to classify particular subclasses of these representations, including connections with the study of endomorphisms of $\mathcal{B}(\mathcal{H})$, finitely correlated states, dilation theory, wavelet analysis, and the theory of non-self-adjoint operator algebras (see $[\mathbf{3}, \mathbf{4}, \mathbf{1 0}, \mathbf{1 1}, \mathbf{1 5}]$ for examples from different perspectives).

The representations $\pi$ of $\mathcal{O}_{n}$ on a space $\mathcal{H}$ which are of interest here have the property that there exists a finite-dimensional subspace $\mathcal{K}$ of $\mathcal{H}$ which is co-invariant and cyclic for the isometries $S=\left(S_{1}, \ldots, S_{n}\right)$, where $\pi\left(s_{i}\right)=S_{i}$. In other words,

(i) $S_{i}^{*} \mathcal{K} \subseteq \mathcal{K}$ for $1 \leqslant i \leqslant n$; and

(ii) $\mathcal{H}=\bigvee_{i_{1}, \ldots, i_{k}} S_{i_{1}} \ldots S_{i_{k}} \mathcal{K}$, where the closed span is over all indices $1 \leqslant i_{1}, \ldots, i_{k} \leqslant n$ and $k \geqslant 1$.

We let $A=\left(A_{1}, \ldots, A_{n}\right)$ be the row contraction of matrices consisting of the compressions to $\mathcal{K}$ of the isometries, so that $A_{i}=\left.P_{\mathcal{K}} S_{i}\right|_{\mathcal{K}}=\left(\left.S_{i}^{*}\right|_{\mathcal{K}}\right)^{*}$. Notice that

$$
\sum_{i=1}^{n} A_{i} A_{i}^{*}=I_{\mathcal{K}}
$$


and thus

$$
\Phi(X)=\sum_{i=1}^{n} A_{i} X A_{i}^{*}
$$

defines a completely positive unital map on $\mathcal{B}(\mathcal{K})$. These representations were classified in [11]. They form the subclass of representations of $\mathcal{O}_{n}$ which arise through the minimal isometric dilations of row contractions of matrices $[\mathbf{6}, \mathbf{1 2}, \mathbf{2 0}]$. They also include the representations of $\mathcal{O}_{n}$ which come from wavelet analysis.

Every orthogonal wavelet of scale $n$ is determined by a scaling function $\varphi$ in $L^{2}(\mathbb{R})$ that determines functions which generate a 'wavelet basis' for $L^{2}(\mathbb{R})$. On the other hand, the Fourier expansion of $\varphi$ also determines so-called wavelet filter functions $m_{1}, \ldots, m_{n}$ in $L^{\infty}(\mathbb{T})$. Let $\rho$ be a primitive $n$th root of unity. The orthogonality of the wavelet is embedded in the statement that the complex matrices $(1 / \sqrt{n})\left(m_{i}\left(\rho^{k} z\right)\right)_{i, k=1}^{n}$ are unitary for a.a. $z \in \mathbb{T}$. Given such a wavelet, a representation of $\mathcal{O}_{n}$ on $L^{2}(\mathbb{T})$ is obtained by defining isometries $S_{i} f(z)=m_{i}(z) f\left(z^{n}\right)$ for $1 \leqslant i \leqslant n$.

Extensive analysis has been conducted on these and other related wavelet representations (see $[\mathbf{3}, \mathbf{4}, \mathbf{1 5}, \mathbf{1 6}]$ for instance). When the scaling function $\varphi$ is compactly supported, the associated representation possesses a finite-dimensional subspace $\mathcal{K}$ which satisfies (i) and (ii) above for the isometries $S_{i}$. In fact, these representations are quite specialized in that $\mathcal{K}$ can be chosen to be spanned by Fourier basis vectors. Thus, the orthogonal wavelet representations form a subclass of the $\mathcal{O}_{n}$ representations arising through dilation theory. Hence, the analysis in $[\mathbf{1 1}]$ can be applied to these representations, and it has been recently by Jorgensen [15].

The decomposition theory of $[\mathbf{1 1}]$ can quickly become computationally cumbersome. This basic problem provided the initial motivation for this paper.

Question 3.1. Given a row contraction $A=\left(A_{1}, \ldots, A_{n}\right)$ of matrices, or equivalently a completely positive map $\Phi$ on finite-dimensional space, is it possible to classify the associated representation of $\mathcal{O}_{n}$ just in terms of $\Phi$, without reference to the $n$ operators $A_{i}$ ?

It was shown in [11] that decomposing these representations, which act on infinitedimensional space, amounts to an exercise in finite-dimensional matrix algebra. This matrix algebra essentially consists of identifying a maximal family of pairwise orthogonal minimal $A_{i}^{*}$-invariant subspaces. Each of these minimal 'anchor' subspaces generates an irreducible subspace for the $S_{i}$, and with this information the representations can be classified. Thus we have the following.

Answer 3.2. Theorem 1.1 answers Question 3.1 in the affirmative. In particular, such anchor subspaces are identified by finding any maximal family of mutually orthogonal minimal projections satisfying $p \leqslant \Phi(p)$, and these subspaces only depend on $\Phi$. Thus all the classification results for these representations in previous papers $[\mathbf{3}, \mathbf{4}, \mathbf{1 1}, \mathbf{1 5}]$ can be restated without reference to the minimal $A_{i}^{*}$-invariant subspaces, only to the minimal projections satisfying this inequality. For instance, this gives a new characterization of irreducibility: one of these representations is irreducible precisely when there is a unique minimal projection $p$ satisfying $p \leqslant \Phi(p)$. 
We show how this new perspective can ease the computational burden by considering an example from each of the dilation and wavelet settings.

Example 3.3. The following example is due to Arveson, and appeared in the seminal paper $[\mathbf{2}]$ as an example of a completely positive map for which the fixed-point set $\mathcal{B}(\mathcal{K})^{\Phi}$ is not an algebra. Nonetheless, it provides a satisfying application of the method introduced here. For $k \geqslant 2$, let $\Phi: \mathcal{M}_{k} \rightarrow \mathcal{M}_{k}$ be the completely positive unital map defined by (assuming an orthonormal basis for $\mathbb{C}^{k}$ has been fixed)

$$
\Phi\left(\left[x_{i j}\right]\right)=\left[\begin{array}{cccc}
x_{11} & & & 0 \\
& \ddots & & \\
& & x_{k-1 k-1} & \\
0 & & & \frac{1}{k-1} \sum_{i=1}^{k-1} x_{i i}
\end{array}\right] .
$$

Without the new perspective discussed above, classifying a representation of $\mathcal{O}_{n}$ generated by $\Phi$ through dilation theory would first require finding a row contraction $A=\left(A_{1}, \ldots, A_{n}\right)$ which determines $\Phi$ as in (1.1). Next, the minimal $A_{i}^{*}$-invariant subspaces would have to be computed. With the new perspective this becomes a triviality: the rank-one projections $E_{1,1}, \ldots, E_{k-1, k-1}$ clearly satisfy $E_{i i} \leqslant \Phi\left(E_{i i}\right)$, and are obviously minimal with respect to this property. This is all the information we need here to describe a representation of $\mathcal{O}_{n}$ generated by $\Phi$, and we emphasize that no reference is required to a row contraction $A$ which determines $\Phi$.

Indeed, it follows that such a representation breaks up into the direct sum of $k-1$ irreducible subrepresentations. In particular, the representation will be irreducible if and only if $k=2$. The ranges of $E_{1,1}, \ldots, E_{k-1, k-1}$ provide one-dimensional 'anchor' subspaces which generate the irreducible subspaces associated with the irreducible subrepresentations. Further, some thought shows that the free semigroup algebra $\mathfrak{S}$ (the WOT (weak operator topology)-closed algebra generated by the isometries from the dilation $[\mathbf{9 - 1 1}]$ ) associated with each subrepresentation is unitarily equivalent to the tractable 'one-dimensional atomic' free semigroup algebra arising in the literature [9]. Thus the free semigroup algebra of the full representation is unitarily equivalent to the direct sum of $k-1$ copies of this algebra.

Example 3.4. Matrix representations were worked out in $[\mathbf{3}]$ for the completely positive maps $\Phi$ determined by wavelet representations of $\mathcal{O}_{3}$ with $\mathcal{K}=\operatorname{span}\left\{z^{0}, z^{-1}, z^{-2}\right\} \subseteq$ $L^{2}(\mathbb{T})$. The authors show how combining an eigenvalue analysis of the matrix, together with a computation of the fixed-point set for the map, can be used to discern information on the representation. The method presented in this section streamlines this analysis, in that it can be used to obtain this information in one fell swoop.

Consider the ordered basis of standard matrix units for $\mathcal{M}_{3}$

$$
\mathbb{B}=\left\{E_{0,0}, E_{-1,-1}, E_{-2,-2}, E_{0,-1}, E_{0,-2}, E_{-1,-2}, E_{-2,-1}, E_{-1,0}, E_{-2,0}\right\},
$$

corresponding to the ordered basis $\left\{z^{0}, z^{-1}, z^{-2}\right\}$ of $\mathcal{K}$. An example of a matrix representation $[\Phi]_{\mathbb{B}}$ in this basis for a completely positive map $\Phi$ determined by a wavelet 
representation of $\mathcal{O}_{3}$ is given by

$$
[\Phi]_{\mathbb{B}}=\left[\begin{array}{ccccccccc}
1 & 0 & 0 & 0 & 0 & 0 & 0 & 0 & 0 \\
0 & \frac{1}{\sqrt{2}} & 1-\frac{1}{\sqrt{2}} & 0 & 0 & 0 & 0 & 0 & 0 \\
0 & \frac{1}{\sqrt{2}} & 1-\frac{1}{\sqrt{2}} & 0 & 0 & 0 & 0 & 0 & 0 \\
0 & 0 & 0 & \frac{1}{\sqrt{2}} & 0 & -\frac{1}{\sqrt{2}} & 0 & 0 & 0 \\
0 & 0 & 0 & \frac{1}{\sqrt{2}} & 0 & -\frac{1}{\sqrt{2}} & 0 & 0 & 0 \\
0 & \frac{1}{\sqrt{2}} & -\frac{1}{\sqrt{2}} & 0 & 0 & 0 & 0 & 0 & 0 \\
0 & \frac{1}{\sqrt{2}} & -\frac{1}{\sqrt{2}} & 0 & 0 & 0 & 0 & 0 & 0 \\
0 & 0 & 0 & 0 & 0 & 0 & 0 & 0 & 0 \\
0 & 0 & 0 & 0 & 0 & 0 & 0 & 0 & 0
\end{array}\right]
$$

(for the reader of [3], this example is generated by taking $g=2, N=3, \lambda_{0}=1$, $\left.\lambda_{1}=(1 / \sqrt{2})=\lambda_{2}\right)$.

The rank-one projection $E_{0,0}$ satisfies $\Phi\left(E_{0,0}\right)=E_{0,0}$ and is clearly minimal with this property. Solving for projections $p=\left(p_{i j}\right)$, with ranges orthogonal to that of $E_{0,0}$ (so $p_{i j}=0$ if $i=0$ or $\left.j=0\right)$, and such that $p \leqslant \Phi(p)$, yields the inequalities

$$
p_{11} \leqslant \frac{p_{11}}{\sqrt{2}}+p_{22}\left(1-\frac{1}{\sqrt{2}}\right) \quad \text { and } \quad p_{22} \leqslant \frac{p_{11}}{\sqrt{2}}+p_{22}\left(1-\frac{1}{\sqrt{2}}\right) .
$$

Hence $p_{22} \geqslant p_{11}$ and $p_{11} \geqslant p_{22}$, so that equality is achieved. Further elementary analysis shows that $p_{12}=p_{21}=0$. Thus $\Phi\left(E_{-1,-1}+E_{-2,-2}\right)=E_{-1,-1}+E_{-2,-2}$ and this ranktwo projection is minimal satisfying $p \leqslant \Phi(p)$ since any smaller projection satisfying this inequality would also have to satisfy the above inequalities involving $p_{11}$ and $p_{22}$.

It now follows that the associated wavelet representation has two irreducible subrepresentations, and the corresponding irreducible subspaces are generated by the anchor subspaces

$$
\left(E_{-1,-1}+E_{-2,-2}\right) \mathcal{K} \text { and } \quad E_{0,0} \mathcal{K} .
$$

Also, $\mathcal{O}_{3}$ cyclic vectors for the corresponding irreducible summands can be obtained simply by taking bases for the generating anchor subspaces. In particular, $\left\{z^{0}\right\}$ and $\left\{z^{-1}, z^{-2}\right\}$ will suffice for the two subspaces.

\section{Open questions}

We finish by taking the opportunity to pose some open problems motivated by the work in this paper. 
Question 4.1. As Remark 1.4 points out, the equivalences (i)-(iii) in Theorem 1.1 are valid when the $A_{i}$ act on infinite-dimensional space. Thus we ask whether there are subclasses of representations of $\mathcal{O}_{n}$ arising from dilations of infinite-rank $n$-tuples $A$ which can be classified using just the associated completely positive map $\Phi$ ? At present there is not even a non-trivial subclass of such representations which has been classified in any way.

A natural class to consider could be wavelet representations of $\mathcal{O}_{n}$ for which the associated scaling function is not compactly supported. It can be shown that these representations have a co-invariant cyclic subspace $\mathcal{K}$ which is infinite dimensional, hence they do indeed arise from the dilations of infinite-rank $n$-tuples. These representations also have the advantage of having an explicit formula for the generating isometries, which is determined by the wavelet filter functions and the scaling function.

We mention that this class could provide examples that shed light on a deep problem in free semigroup algebra theory, posed in $[\mathbf{1 0}]$, which is related to the invariant subspace problem.

Question 4.2. In many respects, quantum information theory is still in its infancy. For instance, there are certainly connections with operator theory and operator algebras, but these seem to be underdeveloped at present.

A natural question to ask here is whether the representations of $\mathcal{O}_{n}$ determined by quantum channels through dilation theory have a meaningful interpretation in quantum information theory? Could they provide a theory for describing the 'external noise' associated with quantum transmissions?

Question 4.3. We also wonder how the distinguished fixed points $\Phi^{\infty}(p)$ from Theorem 1.1 fit into the analysis of completely positive maps. A connection with eigenvalue analysis is suggested by Lemma 1.3, but we are unable to say anything substantial at this point.

Acknowledgements. This work was partly supported by a Canadian NSERC postdoctoral fellowship.

The author is grateful to Palle Jorgensen and Stephen Power for helpful discussions. Thanks also to members of the Department of Mathematics at the University of Iowa and the Department of Mathematics and Statistics at Lancaster University for kind hospitality during the preparation of this article.

\section{Note added in proof}

We mention that Theorem 2.1 has motivated forthcoming work [14] on quantum error correction. Furthermore, a new simpler proof of Theorem 2.1 in included in [14].

\section{References}

1. G. Amosov, A. Holevo And R. F. Werner, On some additivity problems in quantum information theory, Probl. Inform. Transmission 36 (2000), 25-34.

2. W. Arveson, Subalgebras of $C^{*}$-algebras, II, Acta Math. 128 (1972), 271-308. 
3. O. Bratteli And P. Jorgensen, Wavelet filters and infinite-dimensional unitary groups, in Wavelet Analysis and Applications, Guangzhou, 1999, pp. 35-65; American Mathematical Society/International Press Studies in Advanced Mathematics, vol. 25 (American Mathematical Society, Providence, RI, 2002).

4. O. Bratteli, D. Evans and P. Jorgensen, Compactly supported wavelets and representations of the Cuntz relations, Appl. Computat. Harmon. Analysis 8 (2000), 166-196.

5. O. Bratteli, P. Jorgensen, A. Kishimoto and R. F. Werner, Pure states on $\mathcal{O}_{d}$, J. Operat. Theory 43 (2000), 97-143.

6. J. BunCE, Models for $n$-tuples of non-commuting operators, J. Funct. Analysis $\mathbf{5 7}$ (1984), $21-30$.

7. M. D. Chо , Completely positive linear maps on complex matrices, Linear Algebra Applic. 10 (1975), 285-290.

8. I. ChuANG AND M. NiELSEn, Quantum computation and quantum information (Cambridge University Press, 2000).

9. K. DAVIDSON AND D. PitTs, Invariant subspaces and hyper-reflexivity for free semi-group algebras, Proc. Lond. Math. Soc. 78 (1999), 401-430.

10. K. Davidson, E. Katsoulis And D. PitTs, The structure of free semigroup algebras, J. Reine Angew. Math. 533 (2001), 99-125.

11. K. Davidson, D. KRIBS And M. Shpigel, Isometric dilations of non-commuting finite rank $n$-tuples, Can. J. Math. 53 (2001), 506-545.

12. A. Frazho, Models for non-commuting operators, J. Funct. Analysis 48 (1982), 1-11.

13. J. Glimm, Type I $C^{*}$-algebras, Math. Ann. 73 (1961), 572-612.

14. J. Holbrook, D. Kribs and R. Laflamme, Generalized quantum error correction and the structure of the commutant, in preparation (2003).

15. P. Jorgensen, Minimality of the data in wavelet filters, Adv. Math. 159 (2001), 143-228.

16. P. JoRGENSEN AND D. KRIBS, Wavelet representations and Fock space on positive matrices, J. Funct. Analysis 197 (2003), 526-559.

17. C. KIng, Additivity for a class of unital qubit channels, J. Math. Phys. 43 (2002), 46414653.

18. K. Kraus, General state changes in quantum theory, Ann. Phys. 64 (1971), 311-335.

19. V. PAUlsen, Completely bounded maps and dilations, Pitman Research Notes in Mathematics, vol. 146 (Longman Science Technology, Harlow, 1986).

20. G. POPESCU, Isometric dilations for infinite sequences of noncommuting operators, Trans. Am. Math. Soc. 316 (1989), 523-536.

21. M. B. Ruskai, S. Szarek And E. Werner, An analysis of completely-positive tracepreserving maps on $\mathcal{M}_{2}$, Linear Algebra Applic. 347 (2002), 159-187.

22. P. SHOR, Additivity of the classical capacity of entanglement-breaking quantum channels, Quantum information theory, J. Math. Phys. 43 (2002), 4334-4340. 